\title{
Second-generation antipsychotic long-acting injections: systematic review
}

\author{
W. Wolfgang Fleischhacker
}

\section{Background}

Second-generation antipsychotics (SGAS) represent an advance in the long-term management of schizophrenia.

\section{Aims}

To review the available evidence concerning SGA long-acting injections (LAIS).

\section{Method}

A systematic review of the literature was conducted using PubMed.

\section{Results}

Risperidone long-acting injection was the first licensed SGALAl compound and is effective in the long-term management of schizophrenia, with a safety profile similar to that of oral risperidone. Olanzapine pamoate has recently been approved in Europe. In terms of efficacy, at injection intervals of up to 4 weeks it appears comparable to oral olanzapine, although the potential for 'post-injection syndrome' (delirium) calls for additional safety considerations. Paliperidone palmitate is currently under review with the licensing authorities. It also affords the potential advantage of monthly dosing.

\section{Conclusions}

More long-term comparisons of SGA-LAls with oral SGAs as well as with first-generation antipsychotic LAls are needed. These studies should include cost-effectiveness data.

\section{Declaration of interest}

W.W.F. has received research grants from Janssen-Cilag, Servier, Eli Lilly and BMS/Otsuka. He has received honoraria for educational programmes from Pfizer and AstraZeneca, speaking fees from AstraZeneca, Pfizer, Janssen-Cilag, BMS/ Otsuka and advisory board honoraria from BMS/Otsuka, wyeth, Janssen-Cilag and Astrazeneca.
Second-generation antipsychotics (SGAs) represent an advance in the long-term management of schizophrenia, ${ }^{1}$ particularly regarding extrapyramidal motor safety and subjective tolerability. The increasing popularity of SGAs in the past 15 years has been associated with a decline in the use of first-generation antipsychotic (FGA) long-acting injections (LAIs), as many clinicians and their patients relied on oral SGAs for maintenance treatment. Consequently, many patients were switched from FGA-LAIs to oral SGA drugs - a practice that, interestingly, has never been evaluated scientifically. In the wake of these developments, pharmaceutical companies responded by developing secondgeneration antipsychotic LAI preparations. This article is the first comprehensive review of the available evidence regarding SGA-LAIs.

\section{Method}

PubMed was searched using the keywords antipsychotic, neuroleptic, atypical, second generation, new generation, long acting, depot and the generic names of the currently available antipsychotic drugs. In addition, conference programmes and abstracts of recent scientific meetings were screened and pharmaceutical companies who had presented data at such meetings were contacted for follow-up information.

\section{Results}

The results for each compound are organised in the following order: pre-registration randomised controlled clinical trials, secondary and post hoc analyses from these studies, post-registration evidence pertaining to the treatment of people with schizophrenia, and finally reports dealing with a specific research focus, including imaging and pharmacokinetics.

\section{Risperidone long-acting injection}

At the time of writing, risperidone LAI is one of two licensed SGA-LAIs. As the parent compound risperidone lacks the hydroxyl group traditionally used to bind to an ester facilitating the production of a slow-release mechanism, a delivery system of glycolic acid-lactate copolymer microspheres containing the drug was used. Gradual intramuscular hydrolysis of these microspheres allows a slow release of risperidone.

\section{Registration trials}

The development of injectable risperidone included placebocontrolled trials in which risperidone LAI was compared with oral risperidone, as well as studies in which patients were switched from FGA-LAIs or from oral antipsychotics to risperidone LAI, generally with an observational period of 12 weeks. In addition to these studies, a 1-year open clinical trial, post hoc analyses and prescription audits have been carried out.

In the phase III trials fortnightly injections of risperidone LAI ( $25 \mathrm{mg}, 50 \mathrm{mg}$ and $75 \mathrm{mg}$ ) were evaluated. The $75 \mathrm{mg}$ dose did not confer any advantage in efficacy over the $50 \mathrm{mg}$ dose but did have a higher side-effect burden with extrapyramidal symptoms; the manufacturer therefore did not market the $75 \mathrm{mg}$ dose. It is for this reason that most analyses report results for the two lower doses only.

Patients with schizophrenia $(n=440)$ entered a four-arm, double-blind randomised controlled trial comparing the three doses of risperidone LAI with placebo. ${ }^{2}$ End-point scores on all three doses differed significantly from placebo for Positive and Negative Syndrome Scale (PANSS) scores (Fig. 1). ${ }^{3}$ Higher doses of risperidone LAI were associated with more extrapyramidal symptoms, although these were generally considered mild across dose groups. Both patients and investigators rated injection site pain as low.

In a second double-blind non-inferiority controlled trial (a type of study with the aim of showing that one treatment arm 


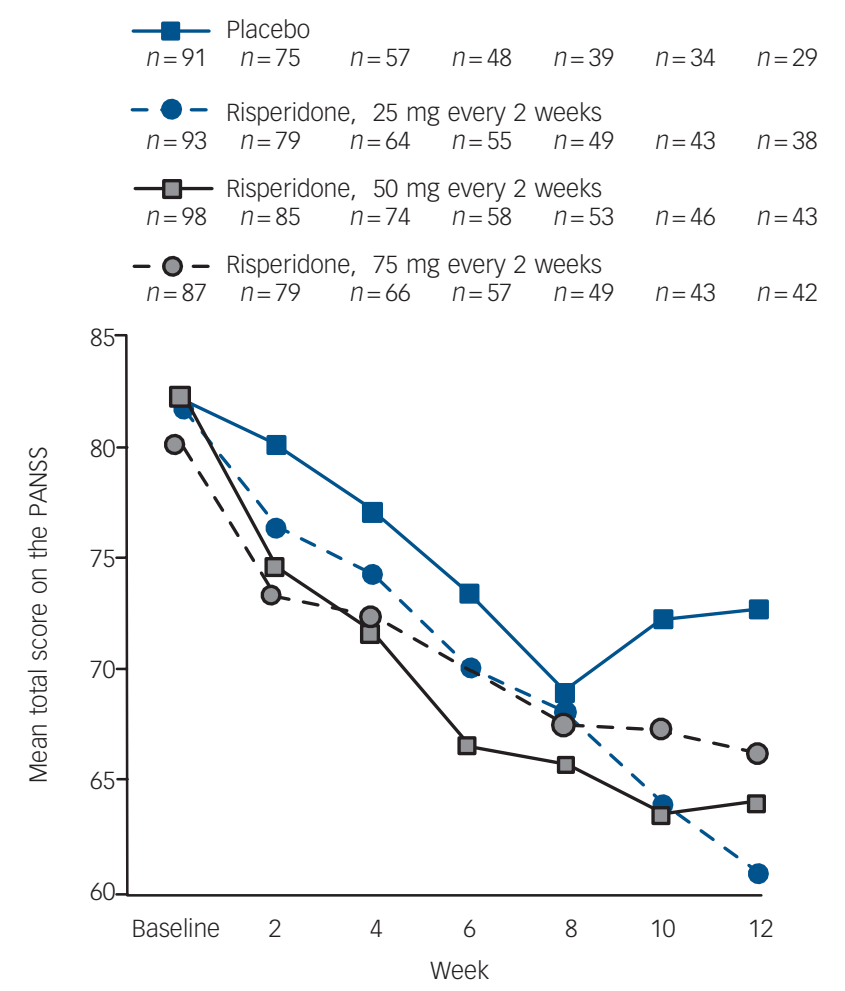

Fig. 1 Change in Positive and Negative Syndrome Scale (PANSS) score over 12 weeks in patients with schizophrenia randomly assigned to double-blind treatment with long-acting injectable risperidone $(25 \mathrm{mg}, 50 \mathrm{mg}$ or $75 \mathrm{mg}$ ) or placebo. Reproduced with permission from Kane et al. ${ }^{2}$

a. Significantly less improvement from baseline seen with placebo than with 50 and $75 \mathrm{mg}$ of risperidone $(P<0.01$ and $P<0.05$ respectively, Dunnett's multiple comparison method).

b. Significantly less improvement from baseline seen with placebo than with $50 \mathrm{mg}$ of risperidone ( $P<0.01$, Dunnett's multiple comparison method).

c. Significantly less improvement from baseline seen with placebo than with 25 and $50 \mathrm{mg}$ of risperidone $(P<0.001$ and $P<0.05$ respectively, Dunnett's multiple comparison method).

d. Significantly less improvement from baseline seen with placebo than with 25 and $50 \mathrm{mg}$ of risperidone $(P<0.001$ and $P<0.05$ respectively, Dunnett's multiple comparison method).

does no worse than another), 640 patients with schizophrenia were prescribed $1-6 \mathrm{mg}$ of oral risperidone for 8 weeks and patients whose condition was stable were then randomised to be treated with risperidone LAI or to continue with oral risperidone. ${ }^{4}$ Both groups demonstrated significant improvement over baseline on PANSS scores and had statistically equivalent efficacy. As in the study by Kane et $a l^{2}$ no unexpected adverse event was observed.

The safety and efficacy of switching from four FGA-LAIs to risperidone LAI (up to $75 \mathrm{mg}$ ) were the main outcome variables of another 12-week study. ${ }^{5}$ Before being switched, patients received two cycles of their original FGA-LAI. Compared with previous treatment, PANSS scores and extrapyramidal symptoms both decreased.

Colleagues and I assessed over 600 patients whose medications were changed from oral risperidone to risperidone LAI over a 1-year open label trial. ${ }^{6}$ Those pre-treated with $1-2 \mathrm{mg}$ of oral risperidone were switched to $25 \mathrm{mg} \mathrm{LAI}$, patients given $2-4 \mathrm{mg}$ received $50 \mathrm{mg}$ LAI and patients originally given $4-6 \mathrm{mg}$ received $75 \mathrm{mg}$ LAI every 2 weeks. Sixty-five per cent of patients completed the full trial, with comparable significant improvements in PANSS scores in all three groups. This study focused on long-term tolerability, and implications for efficacy are limited owing to non-randomised treatment assignment and the lack of a control group.

\section{Secondary and post hoc analyses of registration trials}

A number of secondary analyses have been performed using data from the studies outlined above. It should be emphasised that post hoc analyses have a distinctly different quality compared with studies testing hypotheses, and so results from such studies should be interpreted with caution. The large, 1-year open label trial, referred to above, ${ }^{6}$ was subjected to a number of post hoc analyses as outlined below.

(a) Participants were grouped according to prior antipsychotic: FGA-LAI, ${ }^{7}$ oral $\mathrm{FGA}^{8},{ }^{8}$ or oral risperidone. ${ }^{9}$ Improvements in PANSS scores and extrapyramidal symptoms were evident for all but were most compelling for those switched from stable doses of oral risperidone to risperidone LAI. These results need to be confirmed in prospective clinical trials.

(b) The risk-benefit ratios for patients aged 65 years or older confirmed tolerability and efficacy as seen in younger populations. $^{10}$

(c) Results for participants with schizoaffective disorders $(n=110)$ were detailed separately and showed significant PANSS score improvements. ${ }^{11}$

(d) Five $(0.9 \%)$ of 530 participants with schizophrenia but without dyskinesia at baseline subsequently met predefined tardive dyskinesia criteria. ${ }^{12}$

(e) The main findings of a healthcare resource utilisation analysis were a significant decrease in hospitalisation days and outpatient consultations, which led to the hypothesis that risperidone LAI would, in the long run, decrease healthcare costs for patients with schizophrenia. ${ }^{13}$

(f) Young adults ( $\leqslant 25$ years of age; $n=66$ ) showed no clinically relevant efficacy differences from the older patients, but were more susceptible to experiencing side-effects. ${ }^{14}$

(g) Eighty-five per cent of all patients who had fulfilled symptomatic remission criteria, ${ }^{15-17}$ i.e. a score of $\leqslant 3$ on eight predefined items of the PANSS, at baseline maintained remission status for at least 6 months. Additionally, $21 \%$ of the patients without symptom remission at baseline achieved full remission status by the study end-point. This analysis demonstrated that the concept of remission is clinically testable, and confirms that continuous antipsychotic maintenance treatment has a significant impact on longterm outcome in schizophrenia.

The placebo-controlled trial by Kane et al, ${ }^{2}$ referred to above, was also subjected to post hoc analyses as outlined below.

(a) An analysis of health-related quality of life revealed significant improvements in some aspects of quality of life for patients on active treatment, compared with the placebo group. ${ }^{18}$ Interestingly, patients in the $25 \mathrm{mg}$ dosage group reached normal 36-item Short Form Health Survey (SF-36) values in several domains after only 3 months, and similar results were seen in the 1-year open label trial. ${ }^{6,19}$ These improvements were largely independent of changes in PANSS scores.

(b) With combined use of data from the placebo-controlled study ${ }^{2}$ and the 1-year study, ${ }^{6}$ injection site pain (measured with a $10 \mathrm{~cm}$ visual analogue scale) and patient satisfaction (measured by the Drug Attitude Inventory) ${ }^{20}$ were explored. $^{21}$ Scores were low at baseline with comparable scores for risperidone LAI (all doses) and placebo injections, and pain scores tended to decrease during the two studies. This 
contrasts with notably higher pain scores for four FGA-LAIs in an older study. ${ }^{22}$

(c) Participants from the placebo-controlled study ${ }^{2}$ and a short open label 12-week study ${ }^{23}$ were invited to enrol in an extension study to explore longer-term tolerability. ${ }^{24}$ Mean durations ranged between $347 \quad($ s.d. $=239)$ and 504 (s.d. =505) days and the mean modal dose of risperidone LAI was $50 \mathrm{mg}$ every 2 weeks. Approximately half of both study groups completed 12 months of treatment. Psychosis, headache, insomnia and agitation were the most frequent adverse events. Extrapyramidal symptoms were reported in $22-33 \%$ of patients (proportionally more in the $75 \mathrm{mg}$ dose group).

\section{Post-registration (mostly phase IV) clinical trials}

Randomised controlled clinical trials. A report from Taiwan described a single-blind randomised comparison between daily oral risperidone and risperidone injections $(25 \mathrm{mg}, 37.5 \mathrm{mg}$ or $50 \mathrm{mg}$ ) every 2 weeks in patients pre-treated with oral risperidone (study duration 48 weeks). ${ }^{25}$ Among 50 hospitalised patients with symptomatically stable schizophrenia, $90 \%$ of whom completed the study, PANSS score change was no different between oral risperidone and risperidone LAI groups, but the authors noted a reduced efficacy of LAI at doses below $50 \mathrm{mg}$ every 2 weeks. With regard to safety, patients in the LAI group were reported to experience fewer extrapyramidal symptoms and to have a lower score on the Udvalg for Kliniske Undersøgelser (UKU) side-effect rating scale, as well as lower prolactin levels.

A randomised double-blind 52-week study compared RLAI $25 \mathrm{mg}$ and $50 \mathrm{mg}$ given every 2 weeks. ${ }^{26}$ Only half (51\%) of the 324 randomised patients completed the study. Time to relapse (the primary outcome) was comparable for the two treatment groups, but a modestly lower relapse risk on the higher dose was observed. This also translated into improved psychosocial functioning, again without significant differences between treatment groups. A post hoc analysis of these data revealed that insight was correlated with psychosocial outcomes at end-point. ${ }^{27}$ Change in negative symptoms and duration of LAI treatment also contributed to better psychosocial outcome. Prolactin levels were significantly higher in the $50 \mathrm{mg}$ group at end-point $(50 \mathrm{mg} / \mathrm{l})$.

The most recent addition to the reports of randomised controlled clinical trials of risperidone LAI is a study that compares this treatment with olanzapine tablets. ${ }^{28}$ After data from a large number of patients were excluded from analysis because of protocol amendments and violations, approximately 200 patients receiving a mean dose of $14.6 \mathrm{mg}$ oral olanzapine daily were compared with 155 patients who received a mean modal dose of $40.7 \mathrm{mg}$ risperidone LAI every 2 weeks. There was no substantial group difference in primary and secondary efficacy outcomes, including PANSS scores, Clinical Global Impression (CGI) scores and maintenance of effect. Patients on LAI showed a higher risk of extrapyramidal symptoms and were more often prescribed anticholinergic drugs. The olanzapine group had a greater risk of weight gain and body mass index increase. Two patients died in the risperidone LAI group and six died in the olanzapine group, a fact that the authors do not comment on.

Open, observational and large simple trials. Lindenmayer et al studied symptomatically stable patients with schizophrenia switched from oral haloperidol, quetiapine or olanzapine to risperidone LAI (25-50 mg) and noted small but significant reductions in total PANSS scores by week 12, with switching being well tolerated. ${ }^{23}$
A sample of 1876 patients with schizophrenia or other psychoses were enrolled in a large, open prospective trial, the Switch to Risperidone Microspheres (StoRMi) study, in which patients were switched directly from their previous antipsychotic medication to risperidone LAI $(25-50 \mathrm{mg}) .^{29}$ Three-quarters (74\%) of the sample completed the 6-month trial. Significant improvements in PANSS, CGI, Global Assessment of Functioning, patient satisfaction and quality of life (SF-36) scores were noted. Severity of extrapyramidal symptoms also decreased during the 6-month trial period. Subanalyses of StoRMi have also been conducted and more or less mirror the evidence presented in earlier clinical trials. ${ }^{30}$ Llorca et al followed up 529 StoRMi participants: at 18 months the discontinuation rate was $56 \%$, although $45 \%$ of patients had achieved remission criteria. ${ }^{31}$ Kissling et al noted that four-fifths of the 52 StoRMI participants aged 65 years or over had active concomitant somatic disease. ${ }^{32}$ No patient had had a cerebrovascular adverse event and clinicians judged most of the emerging side-effects as mild or moderate in severity. In patients switching from FGA-LAIs to risperidone LAI a significant reduction in extrapyramidal symptoms was observed.

In a 1-year open label study for clinically stable patients with schizophrenia or schizoaffective disorders, ${ }^{33}$ participants were given two $50 \mathrm{mg}$ doses of risperidone LAI with oral supplementation in the first month and monthly injections thereafter for up to 48 weeks. The intention-to-treat population comprised 67 patients of whom $18 \%$ relapsed. Mean plasma concentrations (risperidone and 9-hydroxyrisperidone) were stable throughout the study period although there appeared to be more variability compared with fortnightly dosing. A dose increase to $75 \mathrm{mg}$ once-monthly in 6-8 participants who had relapsed on $50 \mathrm{mg}$ led to a significant improvement in PANSS total score. The authors cautiously interpret the findings from this pilot trial as encouraging and supportive for 'a possible role for [risperidone] LAI $50 \mathrm{mg}$ once monthly in certain stable patients. ${ }^{33}$ One cannot fail to notice that this trial, although performed 4 years before eventual publication, was never followed up by a controlled trial as suggested by the authors.

Most recently, Emsley et al in 2008 reported results of an open-label trial with risperidone LAI in recent-onset schizophrenia, schizophreniform or schizoaffective disorder. ${ }^{34}$ The sample consisted of people who had met DSM-IV criteria for these disorders for no more than 1 year. Of 50 participants, $72 \%$ completed this 2-year trial and $64 \%$ met remission criteria at the end-point. Improvements were noted in PANSS scores, CGI scores and scales measuring psychosocial outcomes. Most of the patients received either $25 \mathrm{mg}$ or $37.5 \mathrm{mg}$ injections; only $16 \%$ were treated with 2-weekly injections of $50 \mathrm{mg}$ at end-point. Approximately a third of the patients developed extrapyramidal symptoms, including one participant with tardive dyskinesia. As observed in other first-episode samples, ${ }^{35,36}$ there was significant weight gain and body mass index increase in this sample. This underscores the apparent higher likelihood of younger patients developing metabolic side-effects.

Analyses of administrative data, prescription audits and chart reviews. Three reports from UK prescription audits complement the available evidence. Taylor et al evaluated the written prescriptions and reported a significant improvement in CGI scores compared with the pre-treatment period; ${ }^{37}$ they also found a considerable reduction in antipsychotic co-prescriptions. Patel et al attempted to find prognostic indicators for early discontinuation of risperidone LAI and found that patients who were switched from an oral antipsychotic to risperidone LAI were more 
likely to discontinue this drug than those who had previously been treated with an LAI antipsychotic. ${ }^{38}$ Whether this report was derived from the same sample as the one by Taylor et al is not clearly explained. In an extension of these studies, ${ }^{37,38}$ the same group, in a mix of a non-interventional, observational and chart review approaches, evaluated the treatment history of 250 patients who had received prescriptions of risperidone LAI. ${ }^{39}$ Six months after starting treatment $47 \%$ of patients were still receiving this medication. Patients over 55 years old as well as those receiving a dose of more than $25 \mathrm{mg}$ were more likely to continue with risperidone LAI. On the other hand, pre-treatment with clozapine reduced the likelihood of a continuous risperidone LAI prescription.

In another UK study, Niaz \& Haddad compared the total number of in-patient days before and after risperidone LAI prescriptions by reviewing case notes of 90 patients treated for a median duration of 9.5 months. ${ }^{40}$ This mirror-image analysis included 74 patients; 46 patients treated with oral antipsychotics during the same period served as a control group. The latter had significantly lower baseline rates of drug and alcohol misuse as well as fewer forensic problems. On most measures (admissions, compulsory admissions, in-patient days, treatment cost) risperidone LAI was advantageous when compared with previous treatment. The authors critically discuss the results within the limitations of a retrospective case-note study and submit that although they 'cannot prove that risperidone LAI caused the benefits in the primary mirror image analysis, we report an association'. They nevertheless suggest improved adherence to be the most plausible explanation for the advantages of risperidone LAI. A study of similar sample size and design yet with a less sophisticated set of analyses, also performed in the UK, corroborated these findings. ${ }^{41}$

Beneficiaries of Californian Medicaid were investigated in an analysis of administrative data evaluating fluphenazine decanoate, haloperidol decanoate and risperidone LAI. ${ }^{42}$ A mirror-image design comparing the utilisation of these three drugs was adopted, and the sample was composed of large numbers of people treated with the two FGA-LAIs (fluphenazine $n=948$, haloperidol $n=1631)$ and 116 people treated with risperidone LAI. The authors compared the 180 days before with the 180 days after initiating long-acting antipsychotics. Although the authors do report differences between the three LAIs, these appear somewhat irrelevant from a clinical perspective given that less than $10 \%$ of the patients received injections for the full 180-day observation period, and treatment lasted on average 39-48 days. The authors duly note treatment practice differences between California and Europe, and suggest that higher levels of psychiatric comorbidity within their sample contributed to the higher rates of polypharmacy. Like the authors of the two studies reviewed above, ${ }^{40,41}$ they also acknowledge the limitations of analysing administrative data and medical records. ${ }^{42}$

Pharmacokinetic studies. A number of research groups have specifically investigated the pharmacokinetics of risperidone LAI. Castberg \& Speakset studied drug plasma levels in 30 patients receiving risperidone LAI (12-14 days after the final injection, and no sooner than after the fourth injection) compared with 278 patients given oral risperidone. ${ }^{43}$ Serum concentrations of risperidone increased from $38 \mathrm{~nm} / \mathrm{l}$ ( 2 weeks after $25 \mathrm{mg} / 14$ days) to $148 \mathrm{~nm} / \mathrm{l}(75 \mathrm{mg} / 14$ days $)$ in a dose-dependent manner. The concentration-dose ratio for oral risperidone was much more variable.

A retrospective analysis of a therapeutic drug monitoring programme in Norway found a significant percentage of patients treated with RLAI to have plasma levels below what the authors considered to be an established reference range $(30-120 \mathrm{~nm} / \mathrm{l})$, and they too noted substantial individual variation in serum levels. ${ }^{44}$ One suggested reason for this was faulty injection technique (into fatty rather than muscular tissue). Polypharmacy was common in both Scandinavian studies and must be considered a limitation when interpreting the results, and no account was taken of cigarette smoking, which is known to have an impact on some antipsychotic plasma levels. ${ }^{45}$

A more rigorous bioavailability study of risperidone LAI was conducted by the parent pharmaceutical company. ${ }^{46}$ This 15 -week open label study included 86 participants with schizophrenia whose symptoms were stable. Patients were first stabilised on $2 \mathrm{mg}, 4 \mathrm{mg}$ or $6 \mathrm{mg}$ of oral risperidone for at least 4 weeks and then switched to receive $25 \mathrm{mg}, 50 \mathrm{mg}$ or $75 \mathrm{mg}$ of risperidone intramuscularly every 2 weeks. Plasma levels of the active moiety (risperidone plus the major active metabolite 9-hydroxyrisperidone) and risperidone were prospectively collected. There was a dose-proportional increase in steady-state plasma concentrations of the active moiety. Mean steady-state $C_{\max }$ concentrations of the active moiety were $25-32 \%$ lower after risperidone LAI than after oral risperidone, but this was not the case for $C_{\min }$ concentrations. Based on these data the authors postulate bioequivalence of the oral and intramuscular doses tested, although this clearly needs further study, as also argued by Bai et $a l .{ }^{25}$ Plasma level fluctuations were considerably lower for risperidone LAI and this is believed to enhance the safety/ tolerability characteristics of risperidone. Some preliminary evidence for this is provided by Bai et al, who described reduced side-effects and serum prolactin levels associated with a 'relative stability of serum level metabolites. ${ }^{25}$

Imaging studies. Two studies investigated the pharmacodynamics of risperidone LAI using positron emission tomography (PET). ${ }^{47,48}$ Gefvert et al found steady state of the plasma active moiety of risperidone from the fourth injection onwards, and this was maintained for $4-5$ weeks after the last injection. ${ }^{47}$ Dopamine $\mathrm{D}_{2}$ receptor occupancy evaluated 2 weeks after the fifth injection in eight participants revealed a range of occupancy of $25-83 \%$ in a more or less dose-dependent fashion $(25 \mathrm{mg}, 50 \mathrm{mg}$, $75 \mathrm{mg}$ ). Uchida et al assessed monthly injections of risperidone LAI, examining $\mathrm{D}_{2}$ receptor occupancy using $\left[{ }^{11} \mathrm{C}\right]$ raclopride PET. $^{48}$ After at least three consecutive monthly injections of $50 \mathrm{mg}$ risperidone LAI, seven participants had a PET scan within 4 days of the next scheduled injection. The mean total plasma level of the active moiety was $16.6 \mathrm{ng} / \mathrm{ml}$ and $\mathrm{D}_{2}$ occupancy ranged between $29 \%$ and $82 \%$ with a mean value of $56 \%$. None of the patients relapsed during the study. Thus it may not be necessary to achieve continuous striatal $\mathrm{D}_{2}$ blockade greater than $60 \%$ to maintain efficacy.

Surguladze et al used functional magnetic resonance imaging to compare 16 people receiving risperidone LAI with 16 people treated with various FGA-LAIs. ${ }^{49}$ They found that those given FGA-LAIs had worse performance on various memory tasks, with differing cortical activation patterns particularly in the medial and ventrolateral prefrontal areas, compared with those receiving risperidone LAI and the control participants.

Other studies. Generally, individuals with schizophrenia have higher rates of comorbidity of substance misuse, and a group of such patients was the subject of an open, randomised 6-month clinical trial evaluation of the efficacy of risperidone LAI and zuclopenthixol LAI. ${ }^{50}$ In total 115 patients were randomised to receive one of the two drugs during in-patient treatment. The main 
outcome variable was the number of positive urine tests, but psychopathology and adherence to a specific psychotherapeutic programme were also assessed. At end-point the mean dose of risperidone was $47.2 \mathrm{mg}$ LAI per 15 days with an additional $3.4 \mathrm{mg}$ daily of the oral drug. In the zuclopenthixol group patients received $200 \mathrm{mg}$ LAI every 3 weeks and a mean daily oral dose of the same drug of $15 \mathrm{mg}$. Patients on zuclopenthixol had a significantly higher number of positive urine tests. Risperidone showed advantages over zuclopenthixol in PANSS scores and patients also attended more of the psychotherapeutic sessions. One of the explanations the authors offer is the reduced propensity of SGAs to induce extrapyramidal symptoms and dysphoria, both of which may serve as a motive for people with schizophrenia to misuse substances.

\section{Olanzapine long-acting injection}

Olanzapine LAI is a salt of pamoic acid and olanzapine which is suspended in an aqueous vehicle to be injected in the gluteus muscle. The current proposed recommendations for olanzapine LAI use include the advice that oral supplementation of olanzapine is not required during LAI initiation. This new formulation of olanzapine has been approved by the Committee for Medicinal Products for Human Use (CHMP) for Europe and has been submitted to the Food and Drug Administration (FDA) for registration in the USA. The CHMP adopted a positive opinion in September 2008: the document states the approved indication as 'maintenance treatment of adult patients with schizophrenia sufficiently stabilised during acute treatment with oral olanzapine. ${ }^{51}$ To date, two reports have been fully published and others are available as conference abstracts.

Conforming to FDA requirements, the parent pharmaceutical company conducted an 8-week double-blind placebo-controlled study in which 404 patients with schizophrenia were assigned to receive either $210 \mathrm{mg}$ or $300 \mathrm{mg}$ olanzapine LAI fortnightly, or 405 mg LAI every 4 weeks, or placebo. ${ }^{52}$ The PANSS total score change from baseline to end-point was the primary efficacy measure. Patients with a moderate to high level of symptom severity (mean total PANSS scores at baseline for the four groups were 99-103) were allocated to this clinical trial. They had to be hospitalised for the first 2 weeks, and most of the patients remained in hospital throughout the study. The decrease in PANSS total scores

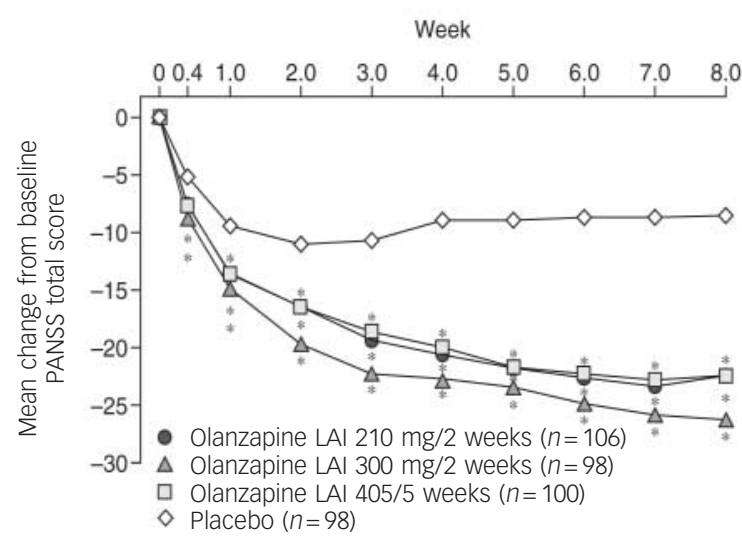

Fig. 2 Weekly mean changes in Positive and Negative Syndrome Scale (PANSS) total score for patients with schizophrenia treated with olanzapine long-acting injection (LAI) or placebo $(n=404)$. Reproduced with permission from Lauriello et al. ${ }^{52}$

${ }^{\star} P<0.05$ type-III sum of squares (analysis of variance). was greater in all olanzapine LAI groups compared with placebo, with no statistically significant difference between the dose groups (Fig. 2). Separation for all three dose groups from placebo occurred at day 7 , the two higher doses separating as early as day $3 .^{52}$

Patients given olanzapine LAI showed significant increases over placebo in fasting total cholesterol and triglyceride levels. Liver function test results were elevated over placebo for the $300 \mathrm{mg}$ olanzapine LAI group. Weight gain was also greater for the olanzapine groups, and amounted to $3.2-4.8 \mathrm{~kg}$ and an incidence of weight gain of $\geqslant 7 \%$ from baseline in the range 3.6-35.4\%. Extrapyramidal symptoms risk did not differ from placebo. Injection site reactions occurred in $3.6 \%$ of olanzapinetreated patients and were described as mild to moderate in severity. Furthermore, no severe sedation was observed in this short study, in contrast to a report of two patients experiencing sedation and delirium in the maintenance study with this preparation. $^{53}$

This trial and other studies were the source of the FDA concerns regarding instances of sometimes profound sedation occurring in the immediate post-injection period addressed at a Psychopharmacologic Drugs Advisory Committee meeting in February 2008. ${ }^{54}$ The CHMP document describes this as a 'post injection syndrome event caused by inadvertent intravenous administration presented with signs and symptoms consistent with olanzapine overdose. ${ }^{51}$ Pending convincing alternative explanations, accidental intravascular administration of olanzapine LAI appears the most likely reason for this syndrome. Theoretically this could also happen even if aspiration shows no blood in the syringe, in cases in which the blood vessel is not directly punctured by the needle but rather indirectly injured by a depot injection in close proximity to a small vessel. In the pre-registration trials, which served as the basis of the submission for marketing authorisation, some patients developed symptoms of sedation and/or delirium. ${ }^{55}$ Initially the incidence per patient was reported as $1.2 \%,{ }^{55}$ but this was later updated to $1.4 \%{ }^{51}$ The injection incidence is 7 per 1000 injections $(0.07 \%)$, based on 25 incidences in 24 patients for 34825 injections. ${ }^{55}$ Other symptoms of post-injection syndrome included extrapyramidal symptoms, dysarthria, ataxia, aggression, dizziness, weakness, hypotension or possible convulsion. In most cases (80\%) initial symptoms appeared within an hour of the injection and full recovery occurred within $24-72 \mathrm{~h}$ after injection. ${ }^{51,55}$ Therefore it is likely that supervised monitoring for $3 \mathrm{~h}$ after injection will be required once this drug is licensed.

Information on the maintenance study has only been published as a conference abstract thus far. ${ }^{53}$ In this trial outpatients with schizophrenia stable on oral olanzapine (10-20 mg per day) for 4-8 weeks were randomised to double-blind treatment with olanzapine LAI $150 \mathrm{mg}$ per 2 weeks $(n=140)$, $300 \mathrm{mg}$ per 2 weeks $(n=141), 405 \mathrm{mg}$ per 4 weeks $(n=318)$ or $45 \mathrm{mg}$ per 4 weeks $(n=144)$. A group of 322 patients continued taking oral olanzapine at their previous dose for 24 weeks. The groups receiving the higher doses of olanzapine LAI were found to be non-inferior to the oral olanzapine group according to their primary outcome measure of exacerbation, defined as hospitalisation or a score above 4 (or an increase of 2 or more points) on any positive item of the Brief Psychiatric Rating Scale. All higher doses were superior to $45 \mathrm{mg}$ olanzapine LAI every 4 weeks, and weight gain was more frequent on the higher doses than on $45 \mathrm{mg}$ and placebo. Two patients experienced post-injection syndrome with sedation and delirium as mentioned above, which was deemed to be due to accidental intravascular injection. Other than this important adverse effect, the safety profile for olanzapine LAI was reported to be largely consistent with that of oral olanzapine. 


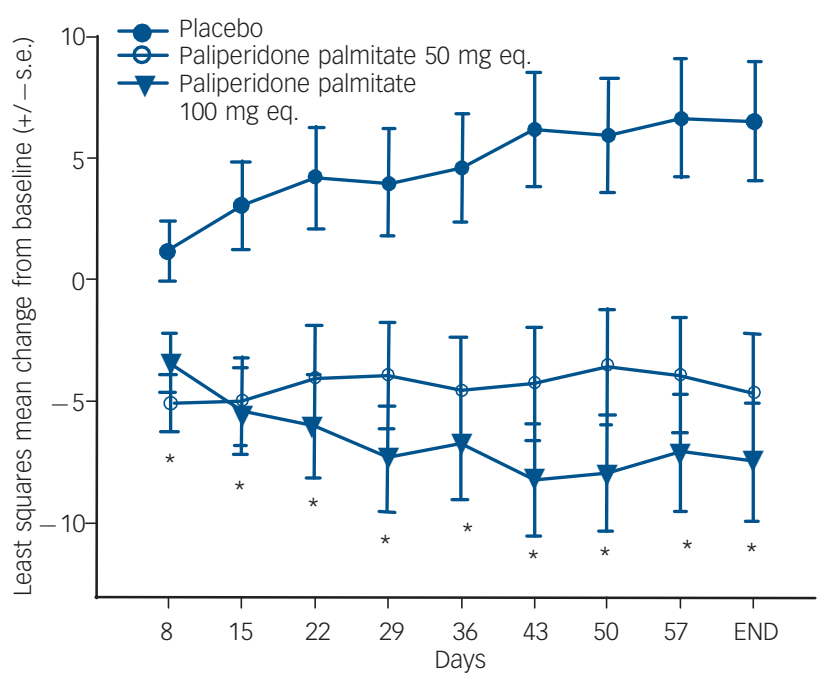

Fig. 3 Changes in Positive and Negative Syndrome Scale (PANSS) scores for patients with schizophrenia treated with paliperidone palmitate. Reproduced with permission from Kramer et al. ${ }^{57}$

That said, oral $v$. LAI dose comparisons and cost-benefit differences between the olanzapine LAI doses require further clarification in future studies.

With regard to imaging studies using $\left[{ }^{11} \mathrm{C}\right]$ raclopride PET, there was $50 \%$ mean striatal $\mathrm{D}_{2}$ receptor occupancy with olanzapine LAI, which was lower than that for oral olanzapine. ${ }^{56}$ Although at the end of the 6-month study $\mathrm{D}_{2}$ occupancy had increased, many patients had received additional oral olanzapine during the study and so these levels have to be interpreted with caution. Overall, olanzapine plasma concentrations showed a good correlation with $\mathrm{D}_{2}$ receptor occupancy.

\section{Paliperidone palmitate}

Currently published information on paliperidone palmitate is only available from conference abstracts. Paliperidone palmitate is an LAI formulation of 9-hydroxyrisperidone, renamed paliperidone. This was studied in a three-arm, randomised double-blind study comparing two doses $\left(50 \mathrm{mg}\right.$ and $100 \mathrm{mg}$ ) with placebo. ${ }^{57}$ Patients were given injections on days 1, 8 and 36. After 9 weeks both doses significantly separated from placebo with regard to PANSS total score and CGI severity ratings (Fig. 3). Insomnia and extrapyramidal symptoms occurred more frequently in the active drug groups than in the placebo group.

A second placebo-controlled study also included a $25 \mathrm{mg}$ dose. ${ }^{58}$ All patients at first received two injections of $50 \mathrm{mg} 1$ week apart and were subsequently given injections every 4 weeks. Doses could be adjusted between $25 \mathrm{mg}, 50 \mathrm{mg}$ and $100 \mathrm{mg}$. If patients reached a PANSS score of $\leqslant 75$ by week 9 they were then randomised (double-blind) to continue either on their previous paliperidone palmitate dose or to the placebo arm in a 24 -week maintenance phase. A preplanned interim analysis included 312 patients and yielded a clear advantage of paliperidone palmitate over placebo. The study was therefore terminated early. Patients given paliperidone palmitate experienced more weight gain but the injection site tolerability was reported to be good.

In another medium-term trial (13 weeks) patients received either one of three doses of paliperidone palmitate $(25 \mathrm{mg}$, $50 \mathrm{mg}, 100 \mathrm{mg}$ ) or placebo in a randomised double-blind study. ${ }^{59}$ Again the first two injections were given a week apart with subsequent 4-week injection intervals. Total PANSS score change from baseline to end-point was superior for all three active drug groups over placebo. Agitation, somnolence, weight increase, dizziness and dry mouth occurred more frequently in paliperidone-treated patients.

Different injection sites (deltoid muscle, gluteal muscle) were compared in a cross-over study with double-blind randomisation to dose $(50 \mathrm{mg}, 75 \mathrm{mg}$ or $100 \mathrm{mg}) .{ }^{60}$ Treatment periods were 13 weeks and 12 weeks respectively. Both injection sites are reported to be equally safe with regard to a comparison of overall treatment-emergent adverse events. Injection site pain was found to be slightly higher with the deltoid injection.

\section{New LAIs in development}

The parent pharmaceutical company for oral aripiprazole has embarked upon a development programme for an aripiprazole LAI formulation. No data are in the public domain as yet. First data on a long-acting injection of iloperidone, yet another putative antipsychotic, has been recently presented. ${ }^{61}$

\section{Discussion}

Second-generation antipsychotic LAIs are an important and developing group of treatment options for people with schizophrenia. Yet despite the potential advantages of LAIs in general over oral medication a number of open questions remain. There are still uncertainties relating to optimal dose as well as the correct injection interval for risperidone LAI for people with schizophrenia. The marketed dose of $37.5 \mathrm{mg}$ has never been subjected to evaluation in a dose-finding study. It would also be helpful to know through randomised studies which doses of risperidone LAI can be used to treat first psychotic episodes and elderly patients. Further data on extending the injection interval beyond 2 weeks should be explored as well.

With regard to olanzapine LAI, it will be interesting to consider whether the potential benefit of a monthly olanzapine injection, as opposed to fortnightly injections with risperidone, outweighs the safety issues of olanzapine concerning monitoring for post-injection syndrome. Also, how would these two drugs perform in a head-to-head comparison study? Could they help in overcoming the stigmatising stereotype that LAIs are only for the most difficult and non-adherent patients?

A number of other SGA-LAIs are currently either under review by regulatory agencies or in development. By increasing the available treatment choices for clinicians and patients alike, new LAI preparations are a welcome development. Clearly, investigators will need to study these preparations beyond the usual registration package. Methodological considerations, particularly for phase IV studies, should include consideration of a priori hypotheses, sufficient power, and enrolment of well-defined representative populations. Moreover, some standardisation or consensus on outcome measures in such studies would be helpful. Also, crucially all studies examining LAIs need to be of sufficient duration to test the underlying hypothesis (e.g. over 1 year for LAI $v$. oral effectiveness). The evidence base concerning comparisons between oral and LAI second-generation antipsychotic preparations is still weak, and long-term head-tohead comparative studies are badly needed. Such studies should include careful evaluation of the economic consequences of the formulation choice. Ideally, comparing the SGA-LAIs with firstgeneration antipsychotic LAIs would be helpful, although the feasibility of this is debatable, given the current reluctance of many patients, relatives and also clinicians to use FGAs. 


\section{Concluding remarks}

Risperidone LAI has been licensed for the past 6 years, yet optimal dose and frequency of injection are still debated and the requirement of initial oral risperidone supplementation remains problematic for some. The high cost of this medication is also an issue. Nevertheless, it has become a useful option for maintenance treatment of psychotic illness. Olanzapine LAI can be given monthly but requires post-injection (supervised) observation for $3 \mathrm{~h}$ in light of the risk of post-injection syndrome. For paliperidone palmitate, only studies of roughly 12 weeks' duration have been reported so far, but these do include reports of a deltoid injection option. Head-to-head comparison studies of sufficient duration are required, as well as comparisons with equivalent oral formulations. Future phase IV studies will require enhanced methodological rigour. Notwithstanding this, an increase in choice of formulation of medication is to be welcomed by clinician and patient alike.

W. Wolfgang Fleischhacker, Professor of Psychiatry, Medical University Innsbruck, Department of Psychiatry and Psychotherapy, Anichstrasse 35, A-6020 Innsbruck, Austria. Email: wolfgang.fleischhacker@i-med.ac.at

\section{References}

1 Tandon R, Fleischhacker WW. Comparative efficacy of antipsychotics in the treatment of schizophrenia: a critical assessment. Schizophr Res 2005; 79: 145-55.

2 Kane JM, Eerdekens M, Lindenmayer JP, Keith SJ, Lesem M, Karcher K. Long acting injectable risperidone: efficacy and safety of the first long-acting atypical antipsychotic. Am J Psychiatry 2003; 160: 1125-32.

3 Kay SR, Fiszbein A, Opler LA. The positive and negative syndrome scale (PANSS) for schizophrenia. Schizophr Bull 1987; 13: 261-76.

4 Chue $\mathrm{P}$, Eerdekens $\mathrm{M}$, Augustyns I, Lachaux B, Molcan $\mathrm{P}$, Eriksson L, et al. Comparative efficacy and safety of long-acting risperidone and risperidone oral tablets. Eur Neuropsychopharmacol 2005; 15: 111-7.

5 Turner M, Eerdekens E, Jacko M, Eerdekens M. Long-acting injectable risperidone: safety and efficacy in stable patients switched from conventional depot antipsychotics. Int Clin Psychopharmacol 2004; 19: 241-9.

6 Fleischhacker WW, Eerdekens M, Karcher K, Remington G, Llorca PM, Chrzanowski W, et al. Treatment of schizophrenia with long-acting injectable risperidone: a 12-month open-label trial of the first long-acting secondgeneration antipsychotic. J Clin Psychiatry 2003; 64: 1250-7.

7 Lasser RA, Bossie CA, Gharabawi GM, Turner M. Patients with schizophrenia previously stabilized on conventional depot antipsychotics experience significant clinical improvements following treatment with long-acting risperidone. Eur Psychiatry 2004; 19: 219-25.

8 Van Os J, Bossie CA, Lasser RA. Improvements in stable patients with psychotic disorders switched from oral conventional antipsychotics therapy to long-acting risperidone. Int Clin Psychopharmacol 2004; 19: 229-32.

9 Lasser RA, Bossie CA, Gharabawi GM, Baldessarini RJ. Clinical improvement in 336 stable chronically psychotic patients changed from oral to long-acting risperidone: a 12-month open trial. Int J Neuropsychopharmacol 2005; 8: 427-38.

10 Lasser RA, Bossie CA, Zhu Y, Gharabawi G, Eerdekens M, Davidson M. Efficacy and safety of long-acting risperidone in elderly patients with schizophrenia and schizoaffective disorder. Int J Geriatr Psychiatry 2004; 19: 898-905.

11 Lasser R, Bossie CA, Gharabawi G, Eerdekens M, Nasrallah HA. Efficacy and safety of long-acting risperidone in stable patients with schizoaffective disorder. J Affect Disord 2004; 83: 263-75.

12 Gharabawi GM, Bossie CA, Zhu Y, Mao L, Lasser RA. An assessment of emergent tardive dyskinesia and existing dyskinesia in patients receiving long-acting, injectable risperidone: results from a long-term study. Schizophr Res 2005; 77: 129-39.

13 Leal A, Rosillon D, Mehnert A, Jarema M, Remington G. Healthcare resource utilization during 1-year treatment with long-acting, injectable risperidone. Pharmacoepidemiol Drug Saf 2004; 13: 811-6.

14 Lasser RA, Bossie CA, Zhu Y, Locklear JC, Kane JM. Long-acting risperidone in young adults with early schizophrenia or schizoaffective illness. Ann Clin Psychiatry 2007; 19: 65-71.
15 Andreasen NC, Carpenter WT, Kane JM, Lasser RA, Marder SR, Weinberger DR. Remission in schizophrenia: proposed criteria and rationale for consensus. Am J Psychiatry 2005; 162: 441-9.

16 Van Os J, Burns T, Cavallaio R, Leucht S, Peuskens J, Helldin L, et al. Standardized remission criteria in schizophrenia. Acta Psychiatr Scand 2006; 113: 91-5.

17 Lasser RA, Bossie CA, Gharabawi GM, Kane JM. Remission in schizophrenia: results from a 1-year study of long-acting risperidone injection. Schizophr Res 2005; 77: 215-27.

18 Nasrallah HA, Duchesne I, Mehnert A, Janagap C, Eerdekens M. Healthrelated quality of life in patients with schizophrenia during treatment with long-acting, injectable risperidone. J Clin Psychiatry 2004; 65: 531-6.

19 Fleischhacker WW, Rabinowitz J, Kemmler G, Eerdekens M, Mehnert A. Perceived functioning, well-being and psychiatric symptoms in patients with stable schizophrenia treated with long-acting risperidone for 1 year. Br J Psychiatry 2005; 187: 131-6.

20 Awad AG, Hogan TP. Subjective response to neuroleptics and the quality of life: implications for treatment outcome. Acta Psychiatr Scand Suppl 1994; 380: $27-32$.

21 Lindenmayer JP, Jarboe K, Bossie CA, Zhu Y, Mehnert A, Lasser RA. Minimal injection site pain and high patient satisfaction during treatment with longacting risperidone. Int Clin Psychopharm 2005; 20: 213-21.

22 Bloch Y, Mendlovic S, Strupinsky S, Altshuler A, Fennig S, Ratzoni G. Injections of depot antipsychotic medications in patients suffering from schizophrenia: do they hurt? J Clin Psychiatry 2001; 62: 855-9.

23 Lindenmayer JP, Eerdekens E, Berry SA, Eerdekens M. Safety and efficacy of long-acting risperidone in schizophrenia: a 12-week, multicenter, open-label study in stable patients switched from typical and atypical oral antipsychotics. J Clin Psychiatry 2004; 65: 1084-9.

24 Lindenmayer JP, Khan A, Eerdekens M, Van Hove I, Kushner S. Long-term safety and tolerability of long-acting injectable risperidone in patients with schizophrenia or schizoaffective disorder. Eur Neuropsychopharm 2007; 17: 138-44.

25 Bai YM, Chen TT, Chen JY, Chang WH, Wu B, Hung $\mathrm{CH}$, et al. Equivalent switching dose from oral risperidone to risperidone long-acting injection: a 48-week randomized, prospective, single-blind pharmacokinetic study. J Clin Psychiatry 2007; 68: 1218-25.

26 Simpson GM, Mahmoud RA, Lasser RA, Kujawa M, Bossie CA, Turkoz I, et al. A 1-year double-blind study of 2 doses of long-acting risperidone in stable patients with schizophrenia or schizoaffective disorder. J Clin Psychiatry 2006; 67: 1194-203.

27 Gharabawi G, Bossie C, Turkos I, Kujawa M, Mahmoud R, Simpson G. The impact of insight on functioning in patients with schizophrenia or schizoaffective disorder receiving risperidone long-acting injectable. J Nerv Ment Dis 2007; 195: 976-82.

28 Keks NA, Ingham M, Khan A, Karcher K. Long-acting injectable risperidone $v$. olanzapine tablets for schizophrenia or schizoaffective disorder. Br J Psychiatry 2007; 191: 131-9.

29 Möller HJ, Llorca PM, Sacchetti E, Martin SD, Medori R, Parellada E. Efficacy and safety of direct transition to risperidone long-acting injectable in patients treated with various antipsychotic therapies. Int Clin Psychopharmacol 2005; 20: $121-30$.

30 Supplement: Progress in modern psychopharmacology. Clinical experience with risperidone long-acting injectable in schizophrenia and schizoaffective disorders. Journal Psychopharm 2005; 19 (5 suppl): 1-40.

31 Llorca PM, Sacchetti E, Lloyd K, Kissling W, Medori R. Long-term remission in schizophrenia and related psychoses with long-acting risperidone: results obtained in an open-label study with an observation period of 18 months. Int J Clin Pharmacol Therap 2008; 46: 14-22.

32 Kissling W, Glue P, Medori R, Simpson S. Long-term safety and efficacy of long-acting risperidone in elderly psychotic patients. Hum Psychopharmacol Clin Exp 2007; 22: 505-13.

33 Gharabawi GM, Gearhart NC, Lasser RA, Mahmoud RA, Zhu Y, Mannaert E, et al. Maintenance therapy with once-monthly administration of long-acting injectable risperidone in patients with schizophrenia or schizoaffective disorder: a pilot study of an extended dosing interval. Ann Gen Psychiatry 2007; 6: 3.

34 Emsley R, Medori R, Koen L, Oosthuizen PP, Niehaus DJH, Rabinowitz J. Longacting injectable risperidone in the treatment of subjects with recent-onset psychosis. J Clin Psychopharmacol 2008; 25: 210-13.

35 Kahn RS, Fleischhacker WW, Boter H, Davidson M, Vergouwe Y, Keet IPM, et al. Effectiveness of antipsychotic drugs in first-episode schizophrenia and schizophreniform disorder: an open randomised clinical trial. Lancet 2008; 371: 1085-97. 
36 Strassnig M, Miewald J, Keshawan M. Weight gain in newly diagnosed first-episode psychosis patients and healthy comparisons: one-year analysis Schizophr Res 2007; 93: 90-8.

37 Taylor DM, Young CL, Mace S, Patel MX. Early clinical experience with risperidone long-acting injection: a prospective, 6-month follow-up of 100 patients. J Clin Psychiatry 2004; 65: 1076-83.

38 Patel MX, Young C, Samele C, Taylor DM, David AS. Prognostic indicators for early discontinuation of risperidone long-acting injection. Int Clin Psychopharmacol 2004; 19: 233-9.

39 Taylor DM, Young C, Patel MX. Prospective 6-month follow-up of patients prescribed risperidone long acting injection: factors predicting favourable outcome. Int J Neuropsychopharmacol 2005; 9: 1-10.

40 Niaz OS, Haddad PM. Thirty-five months experience of risperidone longacting injection in a UK psychiatric service including a mirror-image analysis of in-patient care. Acta Psychiatr Scand 2007; 116: 36-46.

41 Taylor M, Currie A, Lloyd K, Price M, Peperell K. Impact of risperidone long acting injection on resource utilization in psychiatric secondary care. J Psychopharmacol 2008; 22: 128-31.

42 Olfson M, Marcus SC, Ascher-Svanum H. Treatment of schizophrenia with long-acting fluphenazine, haloperidol or risperidone. Schizophr Bull 2007; 33: $1379-87$.

43 Castberg I, Spigset O. Serum Concentrations of risperidone and 9-hydroxyrisperidone after administration of the long-acting injectable form of risperidone. Ther Drug Monit 2005; 27: 103-6.

44 Nesvag $R$, Tanum $L$. Therapeutic drug monitoring of patients on risperidone depot. Nord J Psychiatry 2005; 59: 51-5.

45 Haring $C$, Meise $U$, Humpel C, Saria A, Fleischhacker WW, Hinterhuber H. Dose-related plasma levels on clozapine: influence of smoking behavior, sex and age. Psychopharmacology 1989; 99: 38-40.

46 Eerdekens M, Van Hove I, Remmerie B, Mannaert E. Pharmacokinetics and tolerability of long-acting risperidone in schizophrenia. Schizophr Res 2004 70: 91-100.

47 Gefvert O, Eriksson B, Persson P, Helldin L, Björner A, Mannaert E, et al. Pharmacokinetics and D2 receptor occupancy of long-acting injectable risperidone (Risperdal Consta TM) in patients with schizophrenia. Int J Neuropsychopharm 2005; 8: 27-36.

48 Uchida H, Mamo DC, Kapur S, Labelle A, Shammi C, Mannaert EJL, et al. Monthly administration of long-acting injectable risperidone and striatal dopamine D2 receptor occupancy for the management of schizophrenia. J Clin Psychiatry 2008; 69: 1281-6.

49 Surguladze SA, Chu EM, Evans A, Anilkumar APP, Patel MX, Timehin C, et al. The effect of long-acting risperidone on working memory in schizophrenia. J Clin Psychopharmacol 2007; 27: 560-70.

50 Rubio G, Martinez I, Ponce G, Jimenez-Arriero MA, Lopez-Munoz F, Alamo C. Long-acting injectable risperidone compared with zuclopenthixol in the treatment of schizophrenia with substance abuse comorbidity. Can J Psychiatry 2006; 51: 531-9.

51 Committee for Medicinal Products for Human Use. Summary of Positive Opinion for Zypadhera 2008: Doc. EMEA/CHMP/342542/2008. CHMP, 2008.

52 Lauriello J, Lambert T, Andersen S, Lin D, Taylor CC, McDonnell D. An 8-week, double-blind, randomized, placebo-controlled study of olanzapine long-acting injection in acutely ill patients with schizophrenia. J Clin Psychiatry 2008; 69: 790-9.

53 Detke H, McDonnell D, Kane J, Naber D, Sethuraman G, Lin D. Olanzapine long-acting injection for the maintenance treatment of schizophrenia: a 24-week, randomized, double-blind trial. Schizophr Res 2008; 102 (suppl 2): 1-279.

54 Food and Drug Administration. Psychopharmacologic Drugs Advisory Committee Meeting. FDA, 2008 (www.fda.gov/ohrms/dockets/ac/08/briefing/ 2008-4338b1-01-fda.pdf).

55 McDonnell D, Sorsaburu D, Brunner E, Detke H, Andersen S, Bergstrom R et al. Post-injection delirium/sedation syndrome observed with olanzapine long-acting injection: review of the first 25 events. Eur Neuropsychopharm 2008; 18 (suppl 4): S437-8.

56 Mamo D, Kapur S, Keshawan M, Laruelle M, Taylor CC, Kothare PA, et al. D2 receptor occupancy of olanzapine pamoate depot using positron emission tomography: an open-label study in patients with schizophrenia. Neuropsychopharmacology 2008; 33: 298-304.

57 Kramer M, Litman R, Hough D, Lane R, Lim P, Lin Y, et al. Paliperidone palmitate, a potential long-acting treatment for patients with schizophrenia. Results of a randomized, double-blind, placebo-controlled efficacy and safety study. Int J Neuropsychopharm 2009, in press.

58 Hough D, Gopal S, Vijapurkar U, Lim P, Morozova M, Eerdekens M. Paliperidone palmitate, an injectable antipsychotic in prevention of symptom recurrence in patients with schizophrenia: a randomized, double blind, placebo-controlled study. Biol Psychiatry 2008; 63 (suppl 1): $285 S$.

59 Nasrallah HA, Gopal S, Quiroz JA, Gassmann-Mayer C, Lim P, Eerdekens M, et al. Efficacy and safety of three doses of paliperidone palmitate, an investigational long-acting injectable antipsychotic in schizophrenia. In American Psychiatric Association 2008 Annual Meeting: New Research Abstracts: 176 (NR4-036). APA, 2008 (http://www.psych.org/ am2008newresearch).

60 Gopal S, Lindenmayer JP, Hough D, Melkote R, Lim P, Eerdekens M. Safety and tolerability of the investigational antipsychotic paliperidone palmitate injected in the deltoid or gluteus muscle in patients with schizophrenia. Biol Psychiatry 2008; 63 (suppl 1): 285S.

61 Hill CL, Phadke D, Boyce KM. Four-week iloperidone depot injectable: safety and pharmacokinetic profile in patients with schizophrenia and schizoaffective disorder. In American Psychiatric Association 2008 Annual Meeting: New Research Abstracts 169 (NR4-022). APA, 2008 (http:// www.psych.org/am2008newresearch) 